\title{
Intracaudal Route of Administration
}

National Cancer Institute

\section{Source}

National Cancer Institute. Intracaudal Route of Administration. NCI Thesaurus. Code C38229.

Administration of a drug within the cauda equina. 